\title{
biclustermd: An R Package for Biclustering with Missing Values
}

by John Reisner, Hieu Pham, Sigurdur Olafsson, Stephen Vardeman and Jing Li

\begin{abstract}
Biclustering is a statistical learning technique that attempts to find homogeneous partitions of rows and columns of a data matrix. For example, movie ratings might be biclustered to group both raters and movies. biclust is a current $\mathrm{R}$ package allowing users to implement a variety of biclustering algorithms. However, its algorithms do not allow the data matrix to have missing values. We provide a new $\mathrm{R}$ package, biclustermd, which allows users to perform biclustering on numeric data even in the presence of missing values.
\end{abstract}

\section{Introduction}

Traditional (one-way) clustering (such as with complete-link hierarchical clustering or $k$-means) aims to partition only rows (or columns) of a data matrix into homogeneous subsets. Rows or columns are clustered simply based upon their relational similarity to other observations. Biclustering simultaneously groups rows and columns to identify homogeneous "cells". Biclustering is known to be NP-hard; as such, every existing algorithm approaches this problem heuristically. This methodology was first investigated by Hartigan (1972) but was not given much attention until applied to gene expression data (Cheng and Church, 2000). Today, biclustering is applied across many areas such as biomedicine, text mining, and marketing (Busygin et al., 2008).

For our purposes, we consider rearranging a data matrix to obtain a checkerboard-like structure where each cell is as homogeneous as possible. In this regard, our algorithm has the same goal as spectral biclustering (Kluger et al., 2003), but approaches the problem in a different way. In contrast to clustering with the end goal being a checkerboard-like structure, other techniques have been proposed based on the singular value decomposition (Lazzeroni and Owen, 2002; Bergmann et al., 2003) and others are based on a graph-theoretic approach (Tan and Witten, 2014). Although each technique is different, each has the goal of finding substructure within the data matrix. In Figure 1 we provide a visual suggestion of our biclustering goal. The color scheme represents similar numeric values and our goal is to rearrange the data matrix so that these values form homogeneous cells.
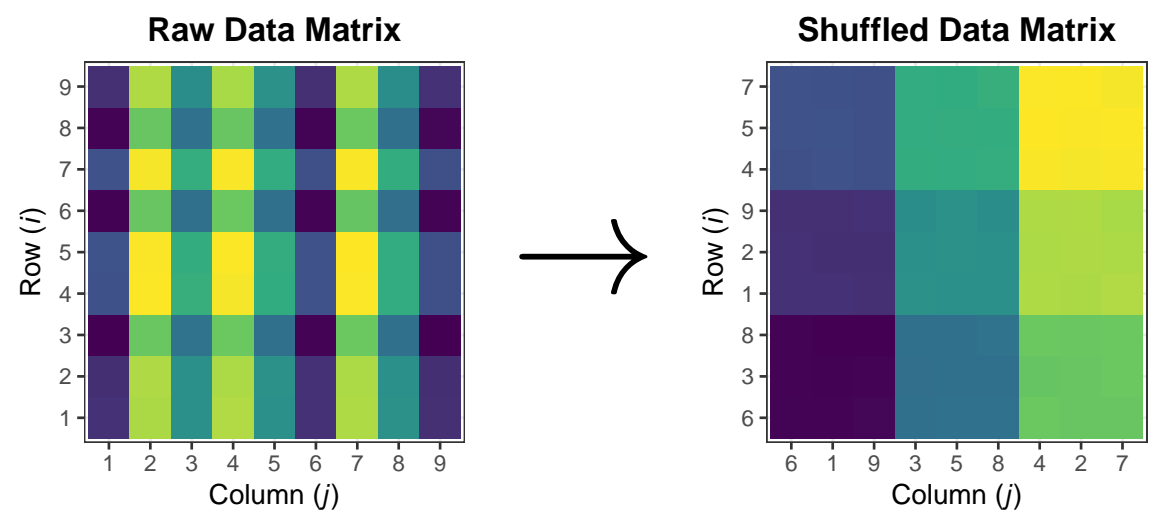

Figure 1: Biclustering with checkerboard-like structure

A publicly available R package for biclustering is biclust by Kaiser and Leisch (2008). This appears to be a commonly used package developed with the intent of allowing users to choose from a variety of algorithms and renderable visualizations. Other biclustering packages include superbiclust, iBBiG QUBIC, s4vd, BiBitR which each provide unique algorithms and implementations (Khamiakova, 2014; Gusenleitner and Culhane, 2019; Zhang et al., 2017; Sill and Kaiser, 2015; Ewoud, 2017). However, from an implementation and algorithmic standpoint, the methods implemented in these packages fail when given a data matrix with missing values. This is clearly a limitation since there exist many rectangular datasets with missing values. For handling missing data, many imputation methods exist in the literature. While this does produce a complete two-way data table, which can subsequently be fully analyzed using existing biclustering algorithms, it has inherent limitations. When large percentages of data are missing, such as is, for example, common in plant breeding and movie rating applications to be discussed later, it is difficult and impossible to reasonably infer missing values. 
Even if a small number of values are missing values those are potentially missing not-at-random due to non-random and unknown devices. For example, in plant breeding, observation may be missing because it is unreasonable to plant a crop in a particular environment or simply because a plant breeder decides to not plant in certain environments. In these cases, imputing missing values would imply that one can confidently estimate the performance of (say) crop yield in an environment where it was never observed growing. There is a large body of literature on the difficult nature of this problem. With this as motivation, our goal was to produce a biclustering algorithm which can successfully deal with data with missing values without applying imputation or making any assumptions about why data are missing.

\section{Biclustering with missing data}

The package described in this paper, biclustermd, implements the biclustering algorithm of Li et al. (2020) and their paper gives a thorough explanation of the proposed biclustering algorithm as well as its applicability. For completeness we give an overview of their algorithm here.

\section{Notation}

- $X$ is a data matrix with $I$ rows and $J$ columns. $X_{i j}$ is a response measure of row $i$ in column $j$ for $i \in\{1,2, \ldots, I\}$ and $j \in\{1,2, \ldots, J\}$.

- Row index set $\mathcal{I}=\{1,2, \ldots, I\}$ is partitioned into $r$ mutually exclusive and exhaustive sets $T_{1}, T_{2}, \ldots, T_{r} . \mathcal{Q} \equiv$ partition of the row index set.

- Column index set $\mathcal{J}=\{1,2, \ldots, J\}$ is partitioned into $c$ mutually exclusive and exhaustive sets $S_{1}, S_{2}, \ldots, S_{c} \cdot \mathcal{P} \equiv$ partition of the column index set.

Our goal for biclustering is to generate a rearranged data matrix with a checkerboard structure such that each "cell" of the matrix defined by $\mathcal{Q}$ and $\mathcal{P}$ is as homogeneous as possible. Depending on specifics of a real problem, "homogeneous" can have different subject matter meanings, and hence optimization of different objective functions can be appropriate. We present our algorithm here with the goal of optimizing a total within-cluster sum of squares given both the row groups in $\mathcal{Q}$ and column groups in $\mathcal{P}$. This can be interpreted as the total sum of squared errors between cell means and data values within cells. Hence we refer to this as SSE. Using the above notations we have $r$ row groups (or row clusters) and $c$ column groups (or column clusters). Let $A$ denote an $r \times c$ "cell-average matrix" with entries

$$
A_{m n} \equiv \frac{1}{\left|\left\{X_{i j}: i \in T_{m} ; j \in S_{n} ; X_{i j} \neq N A\right\}\right|} \sum_{\left\{X_{i j}: i \in T_{m} ; j \in S_{n} ; X_{i j} \neq N A\right\}} X_{i j}
$$

for $m \in 1,2, \ldots, r$ and $n \in 1,2, \ldots, c$. Here, $|\cdot|$ is the set cardinality function and NA denotes a missing value. Then, the within-cluster sum of squares function to be minimized is

$$
\mathrm{SSE} \equiv \sum_{m, n} \sum_{\substack{X_{i j} \neq N A \\ i \in T_{m} \\ j \in S_{n}}}\left(X_{i j}-A_{m n}\right)^{2} .
$$

\section{Biclustering with missing data algorithm}

1. Randomly generate initial partitions $\mathcal{Q}^{(0)}$ and $\mathcal{P}^{(0)}$ with respectively $r$ row groups and $c$ column groups.

2. Create a matrix $A^{(0)}$ using Equation (1) and the initial partitions. In the event that a "cell" $(m, n)$ defined by $\left\{(i, j) \mid i \in T_{m}\right.$ and $\left.j \in S_{n}\right\}$ is empty, $A_{m n}$ can be set to some pre-specified constant or some function of the numerical values corresponding to the non-empty cells created by the partition. (For example, the mean of the values coming from non-empty cells in row $m$ or in column $n$ can be used.) This algorithmic step should not be seen as imputation of responses for the cell under consideration, but rather only a device to keep the algorithm running.

3. At iteration $s$ of the algorithm, with partitions $\mathcal{P}^{(s-1)}$ and $\mathcal{Q}^{(s-1)}$ and corresponding matrix $A^{(s-1)}$ in hand, for $i=1,2, \ldots, I$ let

$$
M_{i n}^{R}=\frac{1}{\left|\left\{j \in S_{n} \mid X_{i j} \neq N A\right\}\right|} \sum_{\substack{j \in S_{n} \\ \text { s.t. } X_{i j} \neq N A}} X_{i j}
$$


for each $n=1,2, \ldots, c$ and compute for $m=1,2, \ldots, r$

$$
d_{i m}^{R}=\sum_{n=1}^{c}\left(A_{m n}-M_{i n}^{R}\right)^{2} \cdot\left|\left\{j \in S_{n} \mid X_{i j} \neq N A\right\}\right| .
$$

Then create $\mathcal{Q}^{(s) *}$ by assigning each row $i$ to $T_{m}$ with minimum $d_{i m}^{R}$.

4. If for $\mathcal{Q}^{(s) *}$ every $T_{m}$ is non-empty, proceed to Step 5. If at least one $T_{m}=\varnothing$ do the following:

(a) Randomly choose a row group $T_{m^{\prime}}$ with $\left|T_{m^{\prime}}\right|>k_{\text {min }}^{R}$ (a user-specified positive integer parameter) and choose $k_{\text {move }}^{R}<k_{\min }^{R}$ row indices to move to one empty $T_{m}$. Choose those indices $i$ from $T_{m^{\prime}}$ with the largest $k_{\text {move }}^{R}$ corresponding values of the sum of squares

$$
\sum_{n=1}^{c} \sum_{\substack{j \in S_{n} \\ \text { s.t. } X_{i j} \neq N A}}\left(X_{i j}-M_{i n}^{R}\right)^{2}
$$

(b) If after the move in (a) no empty row group remains, proceed to Step 5. Otherwise return to (a).

5. Replace $\mathcal{Q}^{(s-1)}$ in Step 3 with the updated version of $\mathcal{Q}^{(s) *}$ and cycle through Steps 3 and $4 \alpha$ times, where $\alpha$ is a user-specified integer parameter. If row_shuffles $>1$, replace $\mathcal{Q}^{(s-1)}$ in 3 . with the updated version of $\mathcal{Q}^{(s) *}$ and cycle through steps 3. and 4. row_shuffles -1 times.

6. Set $\mathcal{Q}^{(s)}=\mathcal{Q}^{(s) *}$. Then update $A^{(s-1)}$ to $A^{(s) *}$ using the partitions $\mathcal{Q}^{(s)}$ and $\mathcal{P}^{(s-1)}$ in Equation (1).

7. For $j=1,2, \ldots, J$ let

$$
M_{j m}^{C}=\frac{1}{\left|\left\{i \in T_{m} \mid X_{i j} \neq N A\right\}\right|} \sum_{\substack{i \in T_{m} \\ \text { s.t. } X_{i j} \neq N A}} X_{i j}
$$

for each $m=1,2, \ldots, r$ and compute for $n=1,2, \ldots, c$

$$
d_{j n}^{C}=\sum_{m=1}^{r}\left(A_{m n}-M_{j m}^{C}\right)^{2} \cdot\left|\left\{i \in T_{m} \mid X_{i j} \neq N A\right\}\right| .
$$

Then create $\mathcal{P}^{(s) *}$ by assigning each column $j$ to $S_{n}$ with minimum $d_{j n}^{C}$.

8. If for $\mathcal{P}^{(s) *}$ every $S_{n}$ is non-empty, proceed to Step 9. If at least one $S_{n}=\varnothing$ do the following:

(a) Randomly choose a column group $S_{n^{\prime}}$ with $\left|S_{n^{\prime}}\right|>k_{\min }^{C}$ (a user-specified positive integer parameter) and choose $k_{\text {move }}^{C}<k_{\text {min }}^{C}$ column indices to move to one empty $S_{n}$. Choose those indices $j$ from $S_{n^{\prime}}$ with the largest $k_{\text {move }}^{C}$ corresponding values of the sum of squares

$$
\sum_{m=1}^{r} \sum_{\substack{i \in T_{m} \\ \text { s.t. } X_{i j} \neq N A}}\left(X_{i j}-M_{j m}^{C}\right)^{2}
$$

(b) If after the move in (a) no empty column group remains, proceed to Step 9. Otherwise return to (a).

9. Replace $\mathcal{P}^{(s-1)}$ in Step 3 with the updated version of $\mathcal{P}^{(s) *}$ and cycle through Steps 7 and $8 \beta$ times, where $\beta$ is a user-specified integer parameter. If col_shuffles $>1$, replace $\mathcal{P}^{(s-1)}$ in 3 . with the updated version of $\mathcal{P}^{(s) *}$ and cycle through steps 7. and 8. col_shuffles-1 times.

10. Set $\mathcal{P}^{(s)}=\mathcal{P}^{(s) *}$ and we have new partitions $\mathcal{Q}^{(s)}$ and $\mathcal{P}^{(s)}$. Then update $A^{(s) *}$ to $A^{(s)}$ using the partitions $\mathcal{Q}^{(s)}$ and $\mathcal{P}^{(s)}$ in Equation (1).

11. Steps 3-10 are executed $N$ times or until the algorithm converges, which is when the Rand Indices for successive row and column partitions are both 1 . (See the description of the Rand Index below.)

Intuitively, our proposed algorithm is nothing more than a rearrangement of rows and columns with the objective to minimize the objectives given in Steps 3 and 7. We consider Step 1 (the random generation of initial cluster assignments) to be of high importance to avoid any bias in the original structure of the data. As a quantitative way to measure the effectiveness of our biclustering, we consider the sum of squared errors (SSE) as the measure of within cell homogeneity. Paired with the SSE, we allow for three different convergence criteria, the Rand Index (Rand, 1971), the Adjusted Rand Index (Hubert and Arabie, 1985), and the Jaccard Index (Goodall, 1966). These indices provide measures for the similarity between two clusterings. 


\section{Overview of biclustermd}

The biclustermd package consists of six main functions with the most important being bicluster(). This function is where the algorithmic process is embedded and contains numerous tunable parameters.

- data: dataset to bicluster. Must be a data matrix/table with only numbers and missing values in the dataset. It should have row names and column names.

- row_clusters: The number of clusters to partition the rows into. Default is $\lfloor\sqrt{I}\rfloor$

- col_clusters: The number of clusters to partition the columns into. Default is $\lfloor\sqrt{J}\rfloor$

- missing_val: Value or function used to represent empty cells of the data matrix. If a value, a random normal variable centered at itself with standard deviation miss_val_sd is used each iteration. Note that this is not data imputation but a temporary value used by the algorithm.

- missing_val_sd: Standard deviation of the normal distribution miss_val follows if miss_val is a number. By default this equals 1 .

- similarity: The metric used to compare two successive clusterings. Can be "Rand" (default), "HA" for the Hubert and Arabie adjusted Rand index or "Jaccard". See clues for details.

- row_min_num: Minimum row cluster size in order to be eligible to be chosen when filling an empty row cluster. Default is $\lfloor I / r\rfloor$.

- col_min_num: Minimum column cluster size in order to be eligible to be chosen when filling an empty column cluster. Default is $\lfloor J / c\rfloor$.

- row_num_to_move: Number of rows to remove from the sampled cluster to put in an empty row cluster. Default is 1 .

- col_num_to_move: Number of columns to remove from the sampled cluster to put in an empty column cluster. Default is 1 .

- row_shuffles: Number of times to shuffle rows in each iteration. Default is 1 .

- col_shuffles: Number of times to shuffle columns in each iteration. Default is 1 .

- max.iter: Maximum number of iterations to let the algorithm run.

- verbose: Logical. If TRUE, will report iteration progress.

In the following sections, we provide an overview of the functionality of biclustermd. For the first dataset, we display the array of visualizations available, in the second example we demonstrate the impact of numerous tunable parameters, our final example demonstrates the computational times of our algorithm.

\section{Example with NYCflights13}

For a first example, we will utilize the flights dataset from Wickham's package nycflights13 (Wickham, 2017). Per the package documentation, flights contains data on all flights in 2013 that departed NYC via JFK, LaGuardia, or Newark. The variables of interest are month, dest, and arr_delay these are the rows, columns and response value, respectively. In a dataset such as this, an application of biclustering would be to determine if there exist subsets of months and airports with similar numbers of delays. From a pragmatic perspective, this discovery may allow for air officials to investigate the connection between these airports and months and why delays are occurring.

Using functions from tidyverse (Wickham, 2016), we generate a two-way data table such that rows represent months, columns represent destination airports, and the numeric response values are the average arrival delays in minutes. This data matrix contains 12 rows (months), 105 columns (destination airports), and approximately $11.7 \%$ missing observations. Below is a snippet of our data matrix.

$\begin{array}{lrrrrr}\text { flights[1:5,1:5] } & & & & \\ & \text { ABQ } & \text { ACK } & \text { ALB } & \text { ANC } & \text { ATL } \\ \text { January } & \text { NA } & \text { NA } & 35.17460 & \text { NA } & 4.152047 \\ \text { February } & \text { NA } & \text { NA } & 17.38889 & \text { NA } & 5.174092 \\ \text { March } & \text { NA } & \text { NA } & 17.16667 & \text { NA } & 7.029286 \\ \text { April } & 12.222222 & \text { NA } & 18.00000 & \text { NA } & 11.724280 \\ \text { May } & -6.516129 & 3.904762 & 10.19643 & \text { NA } & 8.187036\end{array}$


The first step is to determine the number of clusters for months and the number of clusters for destination airports. Since we are clustering months, in this analysis, choosing $r=4$ row clusters seems reasonable (create a group for each season/quarter of the year). Although this is arbitrary, we choose $c=6$ column clusters. Since this algorithm incorporates purposeful randomness (by row and column cluster initialization), biclustermd() should be run multiple times keeping the result with the lowest sum of squared errors (SSE) since it may be expected that for different initialization one can obtain a different local minimum (Li et al., 2020).

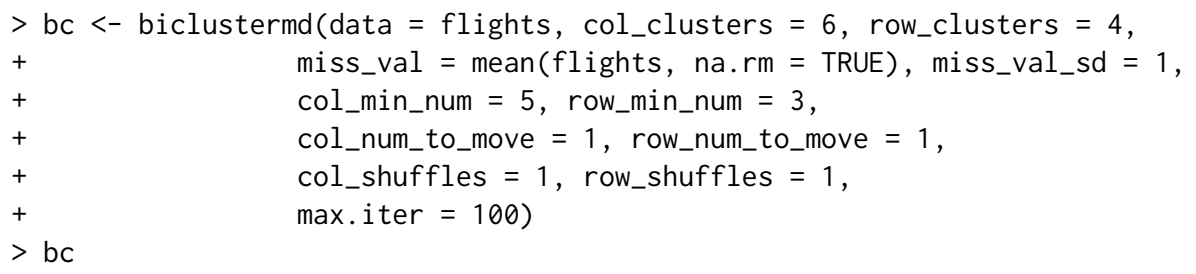

Data has 1260 values, $11.75 \%$ of which are missing

10 Iterations

Initial SSE $=186445 ;$ Final SSE $=82490$

Rand similarity used; Indices: Columns $(P)=1$, Rows $(Q)=1$

The output of biclustermd() is a list of class "biclustermd" and "list" containing the following:

- The two-way table of data provided to the function.

- The final column and row partition matrices.

- SSE generated from the initial partitioning.

- SSE of each iteration, as an "biclustermd_sse" object.

- Similarity measures for rows and columns for each iteration, as an "biclustermd_sim" object.

- The number of iterations to convergence.

- A table of resulting cell means.

\section{Analyzing the NYCflights13 biclustering}

The list output of biclustermd() is used for rendering plots and to obtain cell information. One such visual aid is a plot of the convergence indices versus iteration, given in Figure 2. From this graphic, we can determine the rate at which convergence occurs for both row and column clusters. Moreover, this provides confirmation that our algorithm can indeed achieve good clusterings along both dimensions. Plotting of the similarity measures and SSE is done with autoplot.biclustermd_sim() and autoplot.biclustermd_sse(), methods added to autoplot() of ggplot2 (Wickham, 2009).
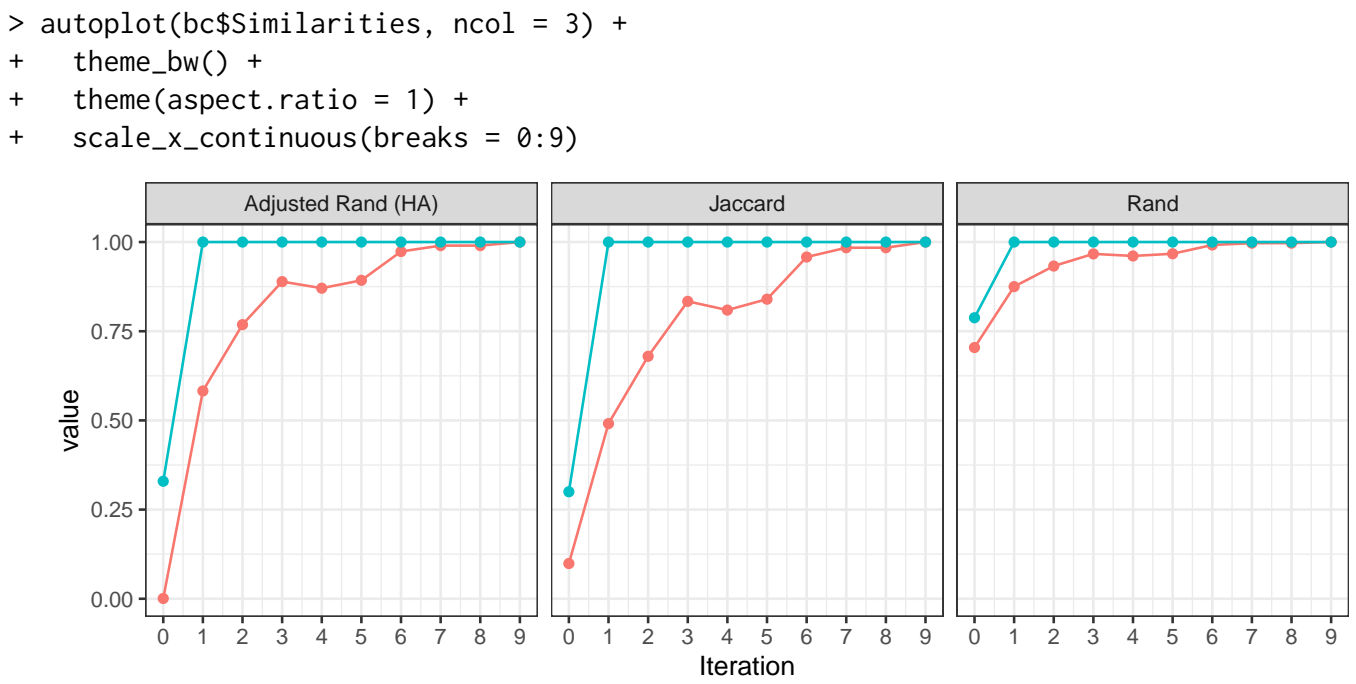

Dimension

$\rightarrow$ Column

$\rightarrow$ Row

Figure 2: Plot of similarity measures for the flights biclustering

In addition to the similarity plots, one can utilize the SSE graphic as an indication of convergence to a (local) minimum biclustering. This can be seen in Figure 3. From this we can observe the rate of 
decrease of the SSE as well as the relative difference between the first and final iteration. Observing closely each of the three convergence criteria suddenly decrease in value along the columns, namely from iteration three to four. The algorithm is simply (attempting to) obtain a lower SSE which may result in column shuffles which differ from iteration to iteration.
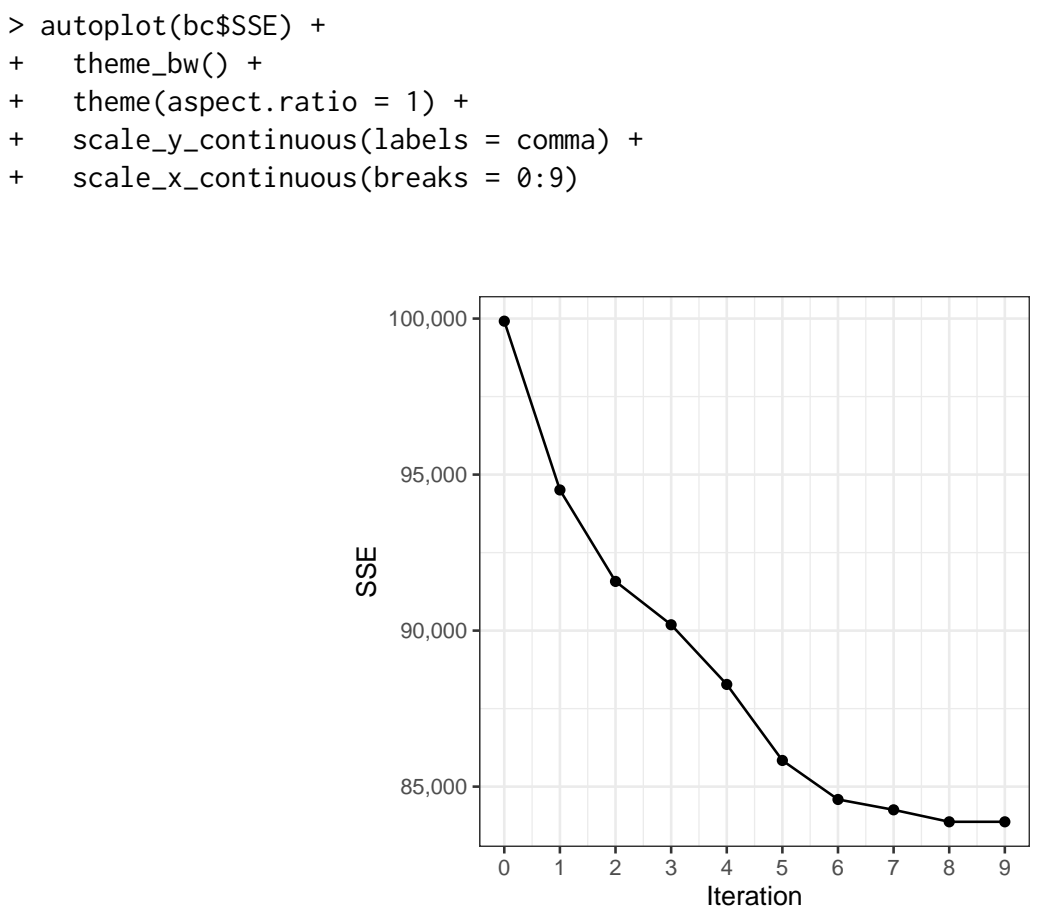

Figure 3: SSE plot of flights biclustering

Traditionally visualizations of biclustering plots are in a heat map fashion. autoplot . biclustermd() makes visual analysis of biclustering results easy by rendering a heat map of the biclustered data and allows for additional customization. Each of Figure 4-7 provide an example of the flexibility of this function. Recall that the algorithm uses purposeful randomness, so a replicated result may look different.

In Figure 4, we provide the default visualization without additional parameters. The white space represent cells without any observations which is directly useful for our interpretation, and the color scale is represented on the same spread as the numerical response.

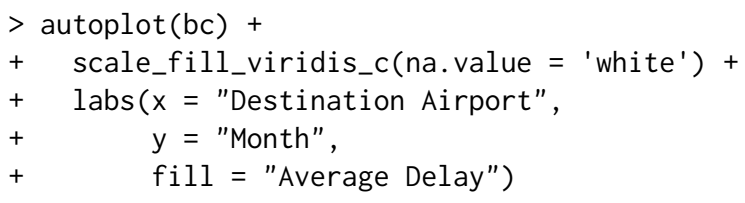

Often it may aid in interpretation to run the data through an $S$-shaped function before plotting. Two parameter arguments in autoplot () are transform_colors = TRUE and $c$ where $c$ is the constant to scale the data by before running it through a standard normal cumulative distribution function. See Figure 5 for an illustration. Applying this transformation, one can immediately notice the distinct dissimilarity between cells that were not clearly present in Figure 4.

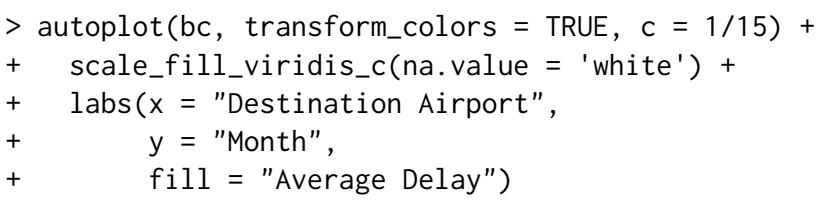

To further aid interpretations, we make use of reorder_biclust in Figure 6. This command reorders row and column clusters from increasing to decreasing mean. In our fights dataset, this may be particularly useful to determine if there is a slow shift in airport locations moving from a high to low number of delays.

$>$ autoplot $(\mathrm{bc}$, reorder $=$ TRUE, transform_colors $=$ TRUE, $c=1 / 15)+$

+ scale_fill_viridis_c $($ na.value $=$ 'white' $)+$ 


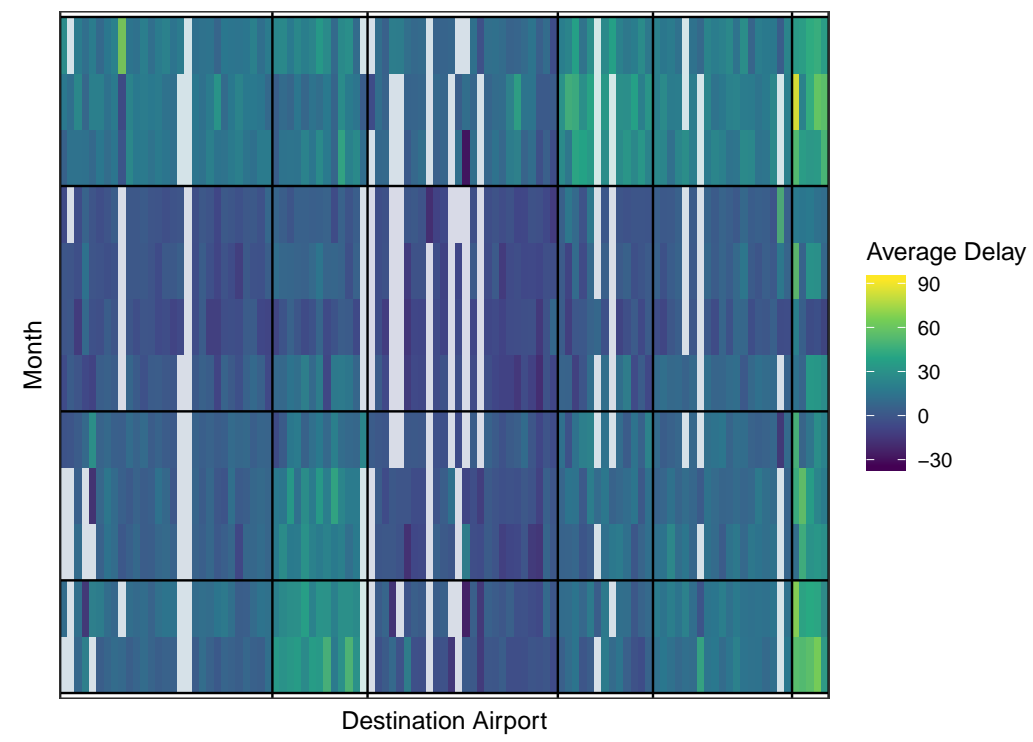

Figure 4: A heat map of the flights biclustering without transforming colors.

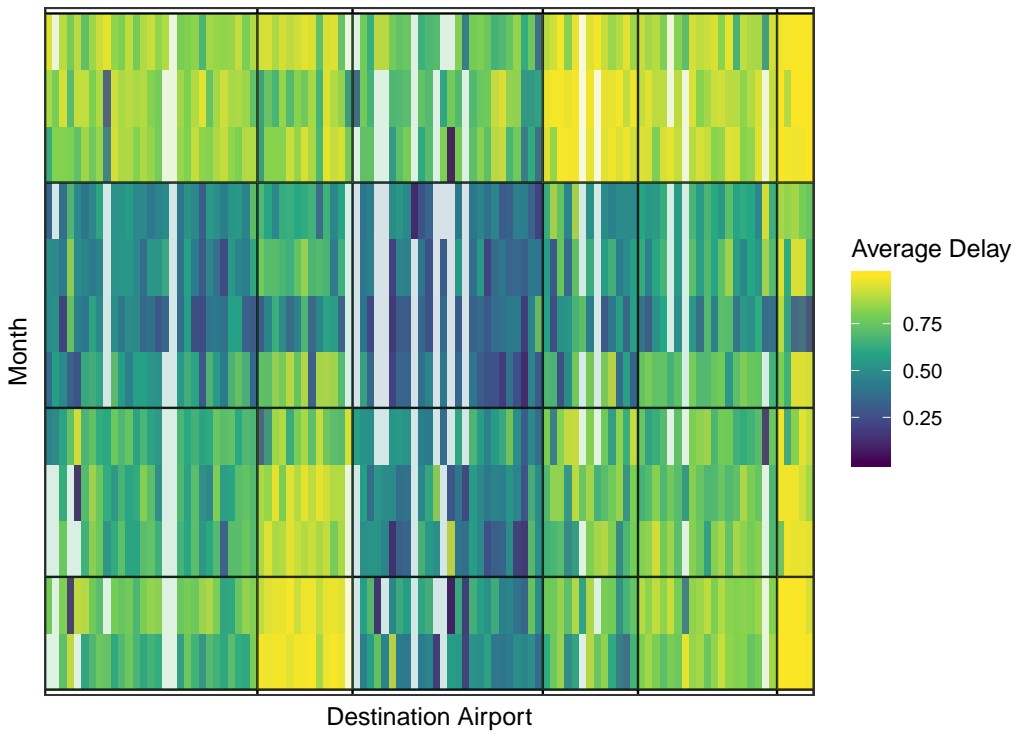

Figure 5: A heat map of the flights biclustering after transforming colors. 


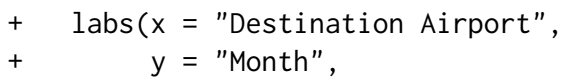

$+\quad$ fill $=$ "Average Delay")

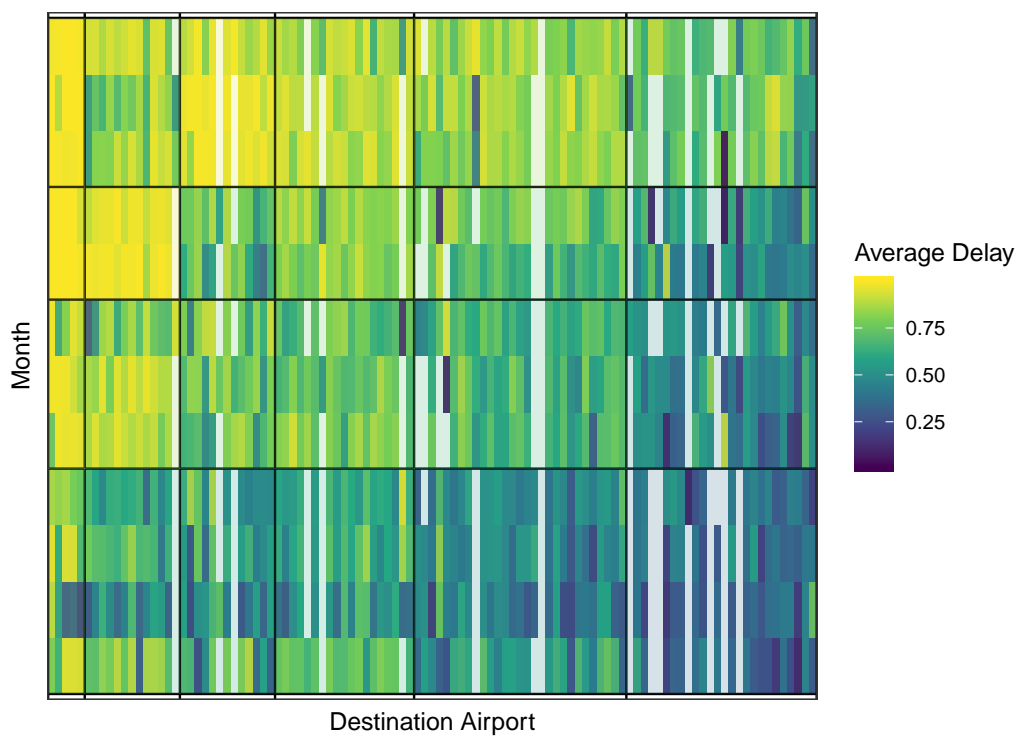

Figure 6: An ordered heat map of the flights biclustering after transforming colors.

Lastly, with large heat maps the authors have found it useful to zoom into selected row and column clusters. In Figure 7, row clusters three and four and column clusters one and four are shown, using the row_clusts and col_clusts arguments of autoplot(). Colors are not transformed.
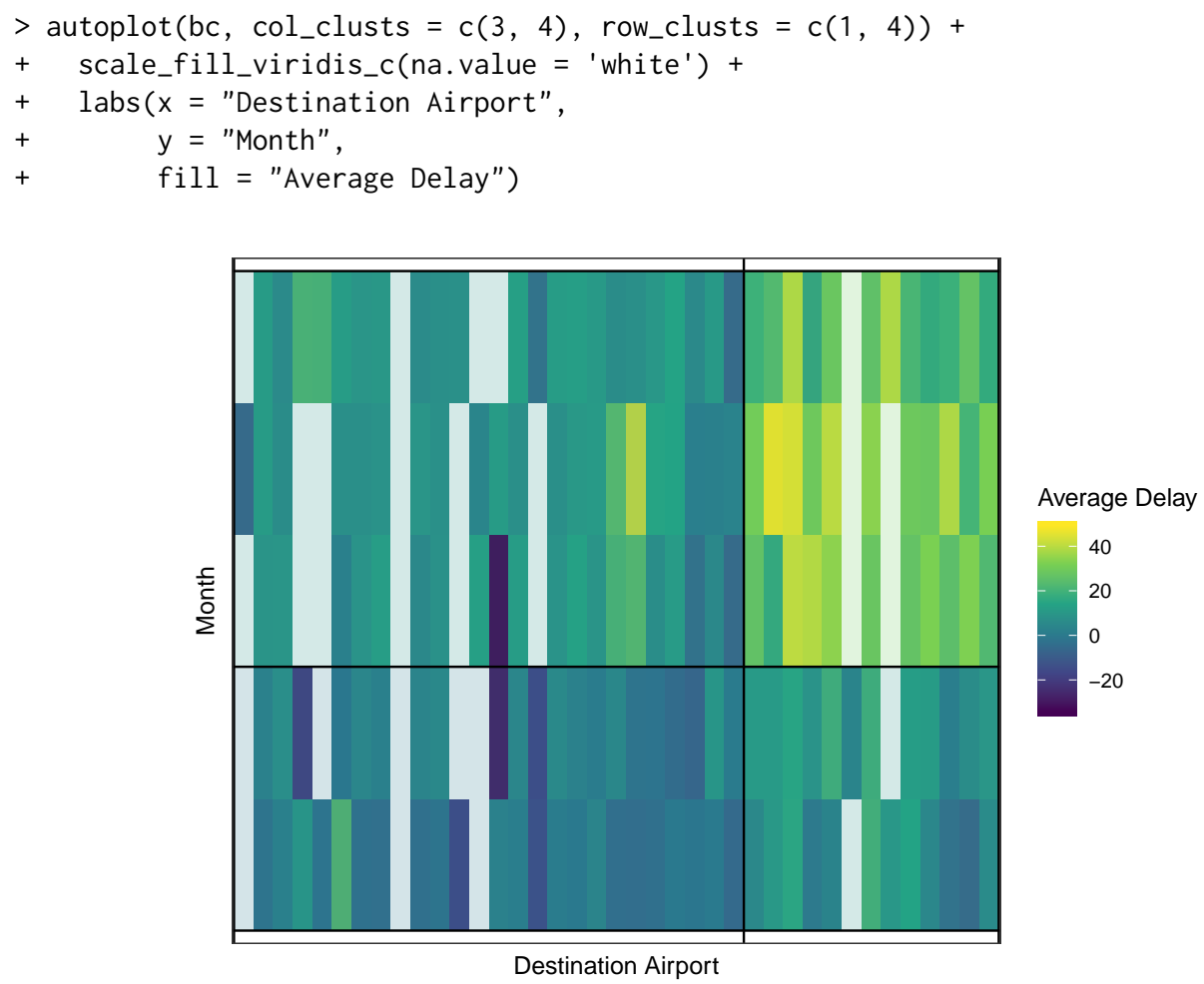

Figure 7: A zoomed in view of the heat map of the biclustering.

There are two additional visualizations that provide insight into the quality of each cell: mse_heatmap() and cell_heatmap(). mse_heatmap() gives the mean squared error (MSE) of each cell. Here, MSE is defined as the mean squared difference between data values and the mean in each cell. Whereas cell_heatmap () provides a heatmap with the total number of observations in the given cell. Combined, these tools provide valuable insight into the homogeneity of each cell. 
$>$ mse_heatmap $(\mathrm{bc})+$

+ theme_bw() +

+ scale_fill_viridis_c() +

$+\operatorname{labs}($ fill $=$ "Cell MSE") +

$+\quad$ scale_x_continuous (breaks $=1: 6$ )

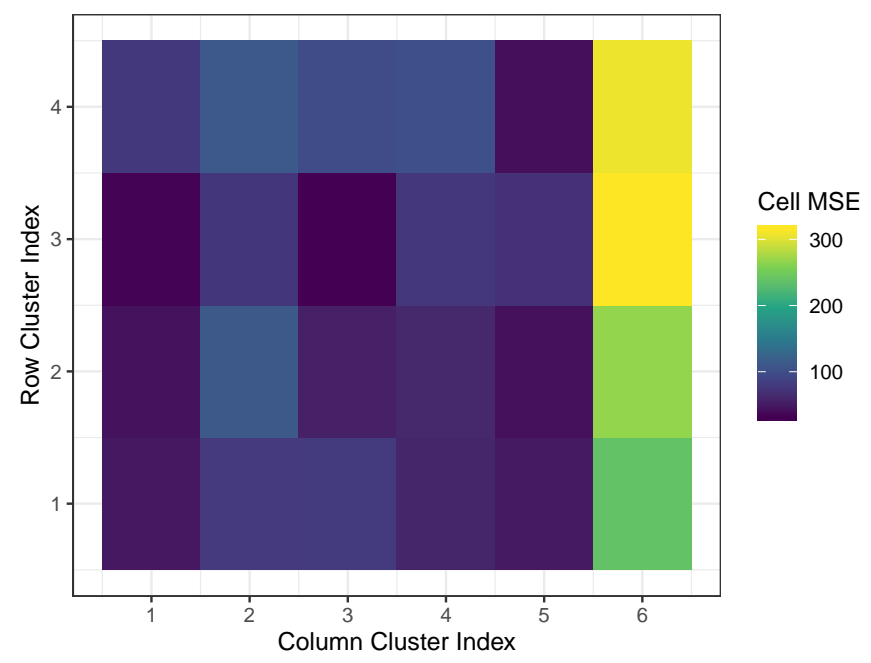

Figure 8: A heat map of cell MSEs for the flights biclustering

$>$ cell_heatmap $(\mathrm{bc})+$

+ theme_bw() +

+ scale_fill_viridis_c()

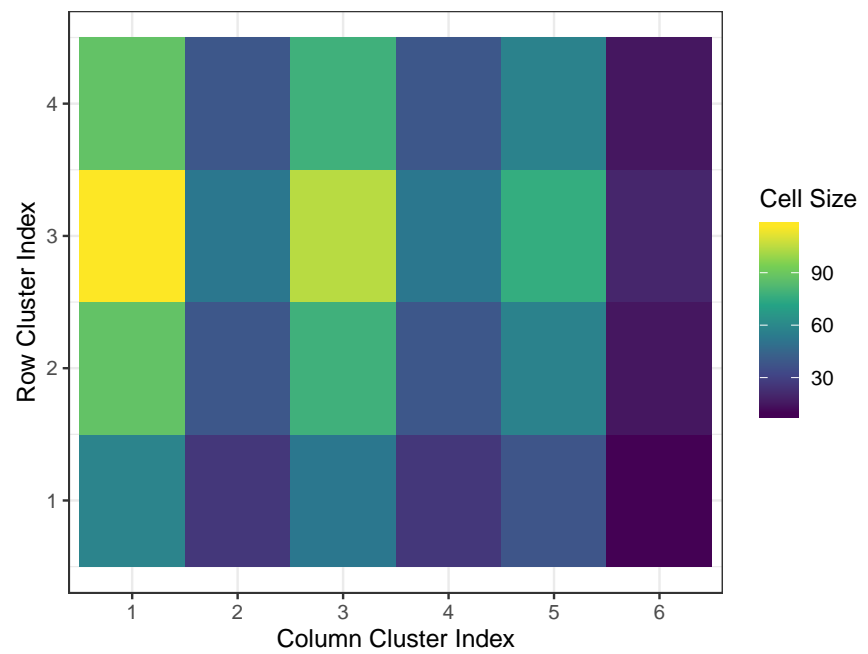

Figure 9: A heat map of cell sizes for the flights biclustering

Finally, for interpretation purposes, retrieving row or column names and their corresponding clusters is easily done using the biclustermd method of row. names() (for rows) and use of a new generic col. names() and its method col. names. biclustermd() (for columns). Two final examples are given below showing the output of each function, which have class data. frame.

$\begin{array}{rrr}> & \text { row.names(bc) } & \%>\text { head() } \\ & \text { row_cluster } & \text { name } \\ 1 & 1 & \text { January } \\ 2 & 1 & \text { April } \\ 3 & 2 & \text { February } \\ 4 & 2 & \text { March } \\ 5 & 2 & \text { August } \\ 6 & 3 & \text { May }\end{array}$




$\begin{array}{rrr}> & \text { col.names (bc) } & \%>\% \text { head() } \\ \text { col_cluster } & \text { name } \\ 1 & 1 & \text { ABQ } \\ 2 & 1 & \text { ACK } \\ 3 & 1 & \text { AUS } \\ 4 & 1 & \text { AVL } \\ 5 & 1 & \text { BGR } \\ 6 & 1 & \text { BQN }\end{array}$

\section{Further capabilities}

As previously mentioned, due to the purposeful randomness of initial row and column clusterings, multiple runs of the algorithm can produce different results. Hence it is recommended to perform several trials (with various parameters) and store the result which obtains the lowest SSE. These multiple runs can easily be done in parallel using the tune_biclustermd() function with the parameters as listed below. To utilize this, first a tuning grid must be defined as an input for tune_biclustermd(). Below we provide an illustration of the process.

- data: Dataset to bicluster. Must to be a data matrix with only numbers and missing values in the data set. It should have row names and column names.

- nrep: dataset to bicluster. The number of times to repeat the biclustering for each set of parameters. Default 10.

- parallel : Logical indicating if the user would like to utilize the foreach parallel backend. Default is FALSE.

- ncores: The number of cores to use if parallel computing. Default 2.

- tune_grid: A data frame of parameters to tune over. The column names of this must match the arguments passed to biclustermd().

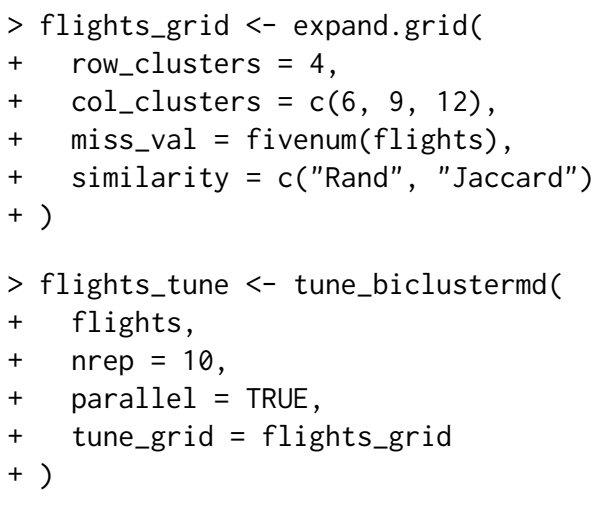

The output of tune_biclustermd() is a list of class "biclustermd" and "list" containing the following:

- best_combn: The best combination of parameters

- best_bc: The minimum SSE biclustering using the parameters in best_combn

- grid: tune_grid with columns giving the minimum, mean, and standard deviation of the final SSE for each parameter combination

- runtime: CPU runtime \& elapsed time.

Users can easily identify which set of tuning parameters gives the best results and corresponding performance with the below code. The minimum SSE is obtained when 12 column clusters are used, the missing value used is -34 , and the Rand similarity is used. A minimum SSE of 70,698 was obtained in the 10 repeats with that combination, which is a $16 \%$ reduction in SSE from our original parameter guesses above. Due to the unsupervised nature of biclustering, ultimately, it is the user's responsibility to choose reasonable number of row and column clusters for interpretations. Each domain and application of biclustering may lead to a different number of desired row or column clusters for a given array size. We simply utilize the SSE and convergence criteria as quantitative measures in determining the quality of the biclustering result.

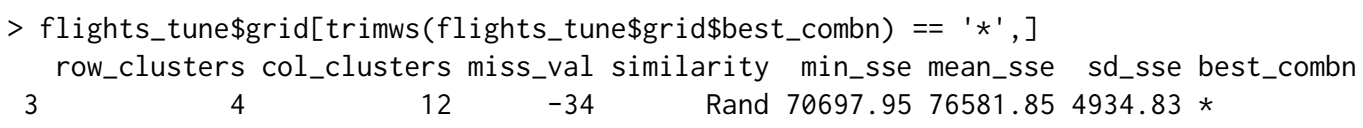


Any of the previously discussed exploratory functions can be used on the biclustering fit with the best tuning parameters by accessing the best_bc element of flights_tune since it is a biclustermd object:

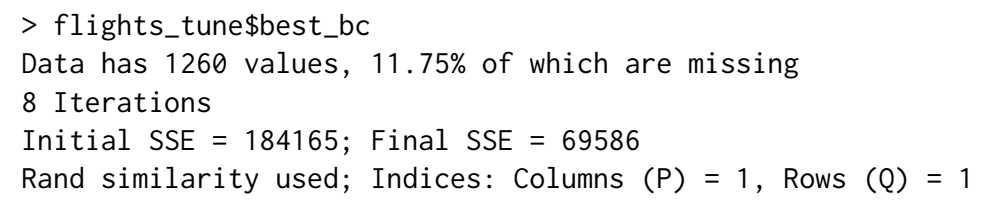

Finally, biclustermd also possesses a method for gather() (Wickham and Henry, 2019) which provides the name of the row and column a data point comes from as well as its corresponding row and column group association. This is particularly useful since we can easily determine the cell membership of each row and column to do further analysis. Namely, given these associations one can further analyze the quality of each cell and paired with domain knowledge of their data make informed judgments about the value of the biclustering. The following output was created from flights_tune\$best_bc.

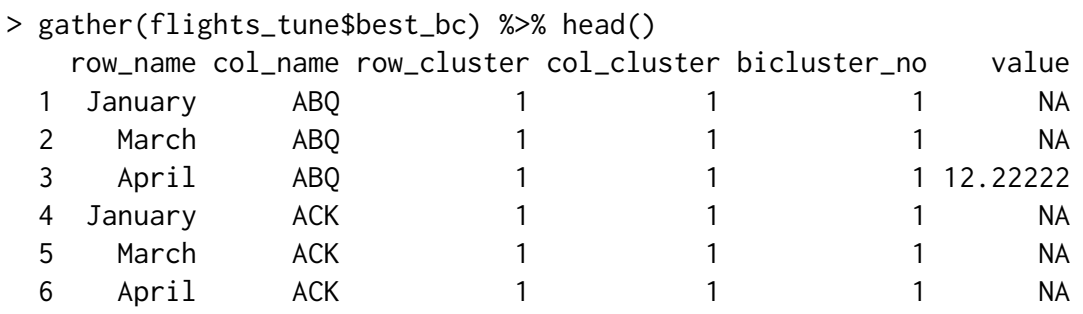

\section{Example with soybean yield data}

For our next example, we perform biclustering on a dataset which has a larger fraction of missing data to further show the practicability of our algorithm. Using data from a commercial soybean breeding program, we consider 132 soybean varieties as rows, 73 locations as columns, and yield in bushels per acre as the response. The locations span across the Midwestern United States and includes parts of Illinois, Iowa, Minnesota, Nebraska, and South Dakota, and each of the 132 soybean varieties represent a different genetic make-up. As one can imagine, not every soybean is grown in each location, as such we obtain a dataset with approximately $72.9 \%$ missing values. One application of a dataset such as this would be to determine if there are some subset of soybeans that perform consistently better (or worse) in some locations than others. From a plant breeding perspective, it is of vital importance to understand the relationship between the genetics and environments of crops, and identifying cells non-overlapping homogeneous cells from biclustering can provide insights into this matter (Malosetti et al., 2013).

The main purpose of this dataset is to demonstrate our algorithm on a dataset with a large amount of missing values as well as show the usefulness of the tuning parameters. Below is our first trial on the soybean yield data where we partition into 10 column clusters, 11 row clusters, and use the Jaccard similarity measure.

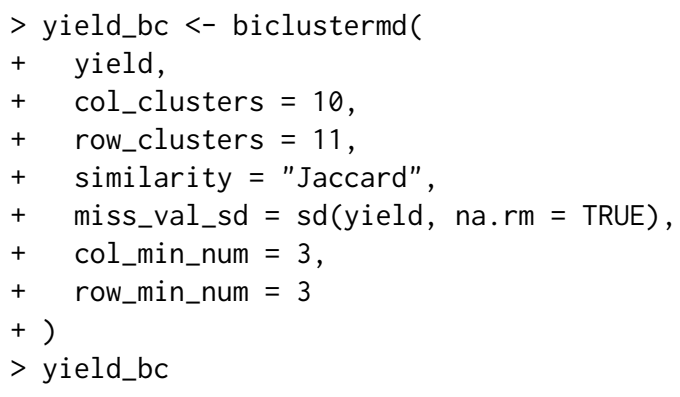

Data has 9636 values, $72.9 \%$ of which are missing

13 Iterations

Initial SSE $=239166 ;$ Final $\mathrm{SSE}=51813$, a $78.3 \%$ reduction

Jaccard similarity used; Indices: Columns $(P)=1$, Rows $(Q)=1$

In observing Figure 10, we notice that perfect convergence through the Rand Index, adjusted Rand Index, and Jaccard similarity; however, the similarities suggest that the columns converge more 
quickly than the rows. This may be attributed to the high percentage of missing values in the rows of the data table. That is, for each location there is more data available than there is for each soybean variety. Again we notice decreases in the values for each of the three indices, but observing Figure 11 , we are assured that the algorithm is only making a column/row swap because a lower SSE is obtainable.
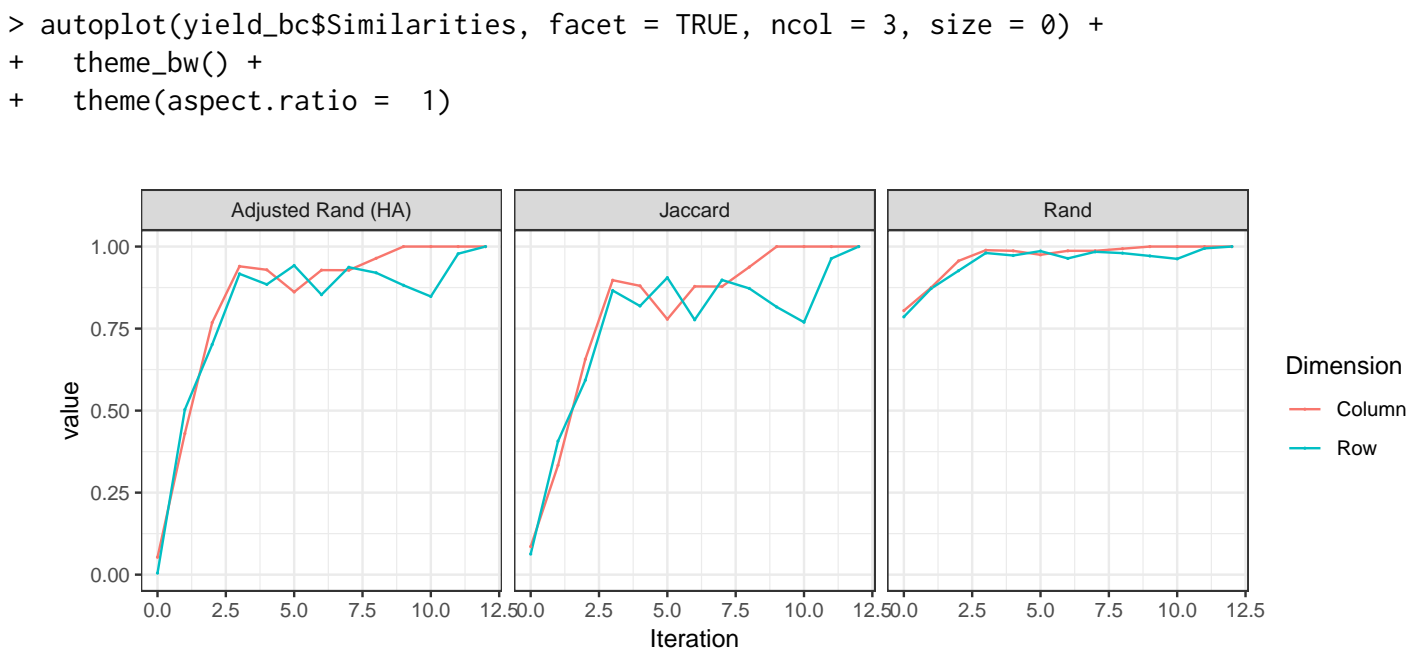

Figure 10: Plot of similarity measures for the soybean yield biclustering
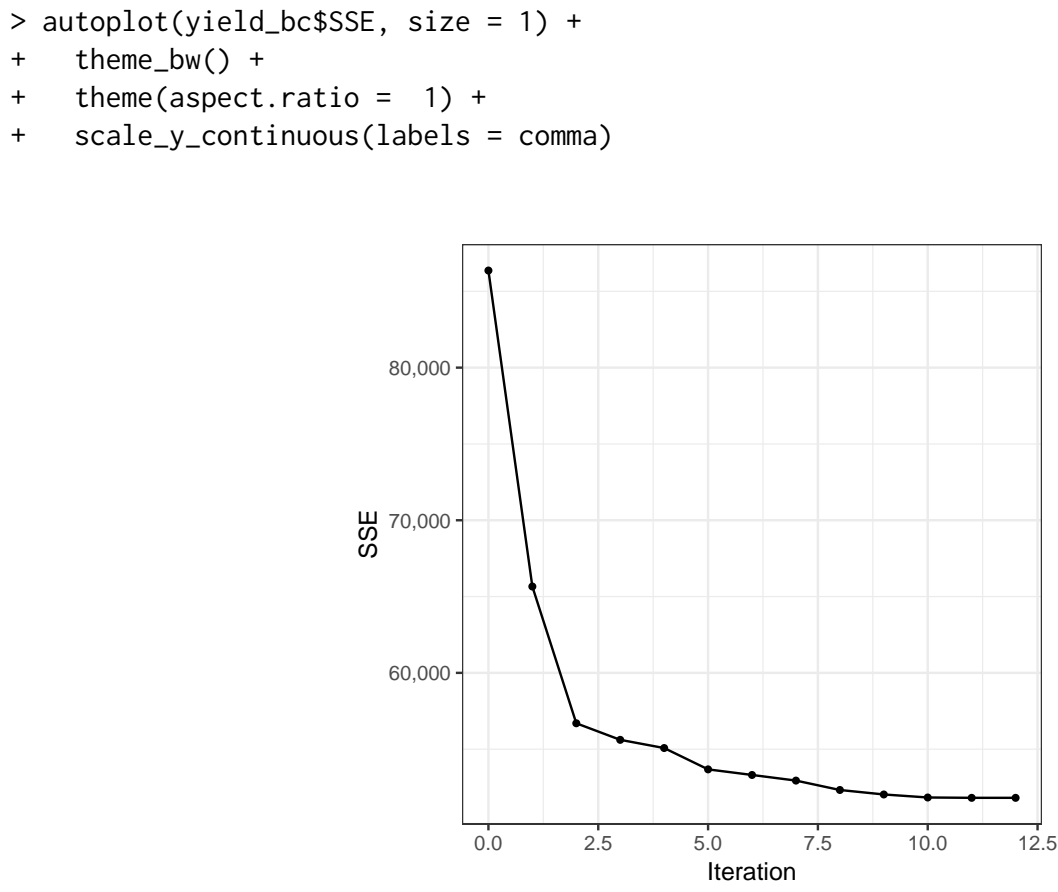

Figure 11: SSE plot of soybean yield biclustering

For the initial trial we observe that the Jaccard index converges in 13 iterations to an SSE value of 51,813 . To see if it is possible to decrease this SSE even further, we test the impact of col_shuffles and row_shuffles. Recall that these parameters determine how many row and column rearrangements the algorithm makes before completing one iteration. Below we use tune_biclustermd() to test combinations of col_shuffles and row_shuffles as well as its corresponding SSE. We define the tune grid to mimic that of the yield_bc creation above, but let col_shuffles and row_shuffles take on values in $\{1,3,6\}$ independent of each other. We repeat the biclustering ten times for each parameter, specified by nrep $=10$. Note that parallel $=$ TRUE allows us to tune over the grid in parallel.

$>$ yield_tbc <- tune_biclustermd (

+ yield, 


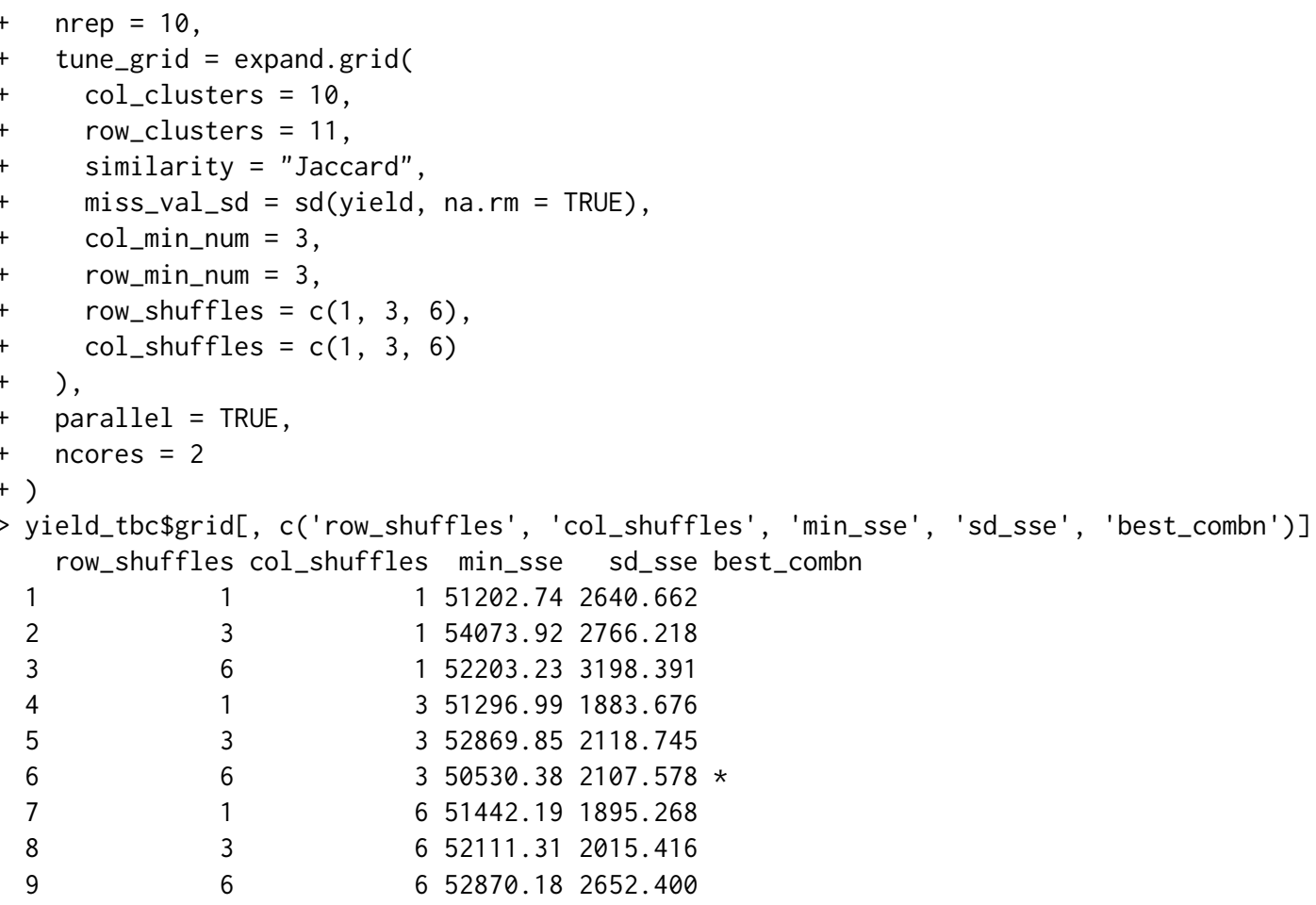

\section{Algorithm time study with movie ratings data}

For our last example, we focus our attention on a movie ratings dataset obtained from MovieLens (Harper and Konstan, 2015). If we consider movie raters as defining rows, movies as defining columns, and a rating from 1-5 (with 5 being the most favorable) as a response, then biclustering can be used to determine subsets of raters who have similar preferences towards some subset of movies.

The main topic of this section will be to perform time studies to test the scalability of our proposed algorithm. In some applications, it is not uncommon to have a two-way data table with 10,000+ rows or columns. Intuitively as the dimensions of the two-way data table increases so will the computational time. In it is not uncommon for other biclustering algorithms to run for 24+ hours (Oghabian et al., 2014). We ran the biclustering over a grid of 80 combinations of $I$ rows, $J$ columns, $r$ row clusters, and $c$ column clusters with 30 replications for each combination. In addition to the four grid parameters, we consider the following metrics which are byproducts of the four parameters: the size of the dataset $N=I \times J$, average row cluster size $I / r$, and average column cluster size $J / c$. Table 1 summarizes the grid parameters, their byproducts and the defined lower and upper limits on each.

\begin{tabular}{lrrrrrrr}
\hline & $N=I \times J$ & $I$ & $J$ & $r$ & $c$ & $I / r$ & $J / c$ \\
\hline Lower Limit & 2,500 & 50 & 50 & 4 & 4 & 5 & 5 \\
Min & 18,146 & 86 & 98 & 4 & 4 & 5 & 5 \\
Mean & 665,842 & 784 & 839 & 42 & 45 & 49 & 47 \\
Max & $1,929,708$ & 1,495 & 1,457 & 239 & 258 & 293 & 346 \\
Upper Limit & $2,225,000$ & 1,500 & 1,500 & 300 & 300 & 375 & 375 \\
\hline
\end{tabular}

Table 1: Summary of the movie data runtime grid with defined lower and upper limits

Table 2 gives a five number summary and the mean runtime in seconds paired with the parameters which produced run times closest to each statistic. In all, we see that the algorithm can take less than a second to run, while in the other extreme the algorithm requires 39 minutes to converge. It is particularly interesting that for the two parameter combinations closest to the median run time, one dataset is nearly twice the size of the other. Furthermore, note than the mean run time is more than twice that of the median, but the size of the dataset is just $38 \%$ of that at the median. However, at the mean, $3744=72 \cdot 52$ biclusters are computed, while at the medians, only $80=20 \cdot 4$ and $481=13 \cdot 37$ biclusters are computed. For a visual summary of the results, we point the reader to Figure 12.

Figure 12 plots run times versus the five parameters controlled for in the study as well as average row cluster size, average column cluster size, and sparsity. We encourage the reader to personally 


\begin{tabular}{lrrrrrrr}
\hline & Seconds & $I$ & $J$ & $N$ & $r$ & $c$ & Sparsity \\
\hline Min & 0.4 & 210 & 98 & 20,580 & 10 & 9 & $96.2 \%$ \\
Q1 & 24.7 & 820 & 184 & 150,880 & 53 & 12 & $98.2 \%$ \\
Median & 63.6 & 988 & 1,240 & $1,225,120$ & 20 & 4 & $98.5 \%$ \\
Median & 63.5 & 501 & 1,302 & 652,302 & 13 & 37 & $98.0 \%$ \\
Mean & 137.5 & 1,084 & 427 & 462,868 & 72 & 52 & $98.4 \%$ \\
Q3 & 141.0 & 485 & 875 & 424,375 & 36 & 126 & $98.0 \%$ \\
Max & $2,369.0$ & 1,495 & 1,233 & $1,843,335$ & 147 & 204 & $98.5 \%$ \\
\hline
\end{tabular}

Table 2: Five number summary and mean runtime in seconds along with parameters achieved at

explore the results; the run time data is the runtimes dataset in the package. Moreover, Li et al. (2020), provides further insights into the effect of sparsity on runtimes.

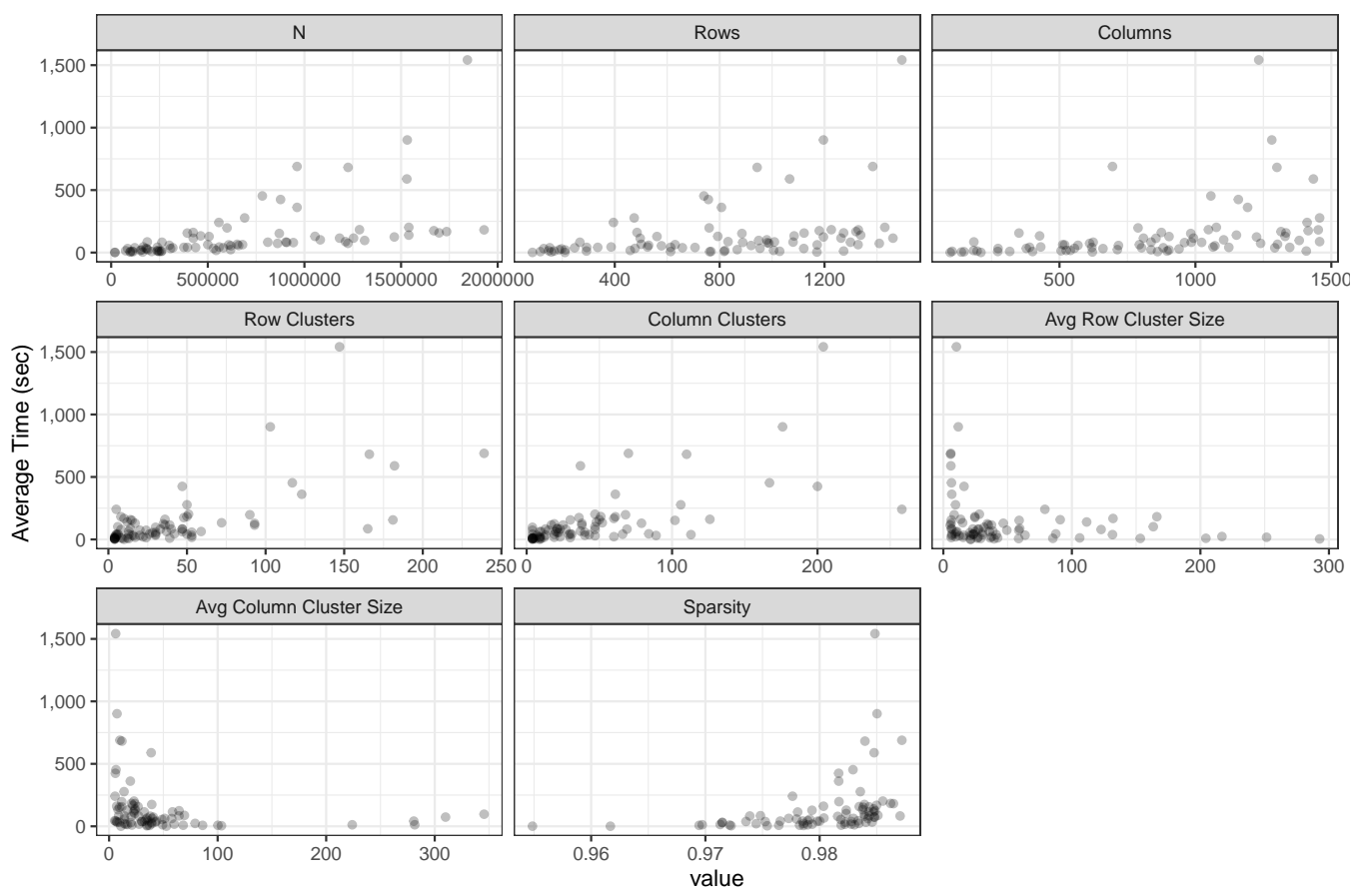

Figure 12: Relationship between movie grid parameters and elapsed time

Finally, we address the trade-off between interpretability and computation time. Figure 13 plots elapsed time versus average cluster size on a doubly log 10 scales for row clusters (left) and column clusters (right). Clearly, computation time can be decreased by increasing the average cluster size, but doing so potentially reduces the interpretability of results; biclusters may be too large for certain use cases. Keeping in mind that the $y$-axis is on a $\log 10$ scale, increasing average cluster size will have diminishing returns. Reviewing the plot on the right-hand side of the second row and the left-hand side of row three in Figure 12 sheds more light into this notion.

\section{Summary}

Based on the work of (Li et al., 2020) we provide a user-friendly $\mathrm{R}$ implementation of their proposed biclustering algorithm for missing data as well as a variety of visual aids that are helpful for biclustering in general and biclustering with missing data specifically. The unique benefit biclustermd provides is in its ability to operate with missing values. Compared to other packages which do not allow incomplete data or make use of some sort of imputation, we approach this problem with a novel framework that does not alter the structure of an inputted data matrix. Moreover, given the tunability of our biclustering algorithm, users are able to run trials on numerous combinations in an attempt to best bicluster their data. 


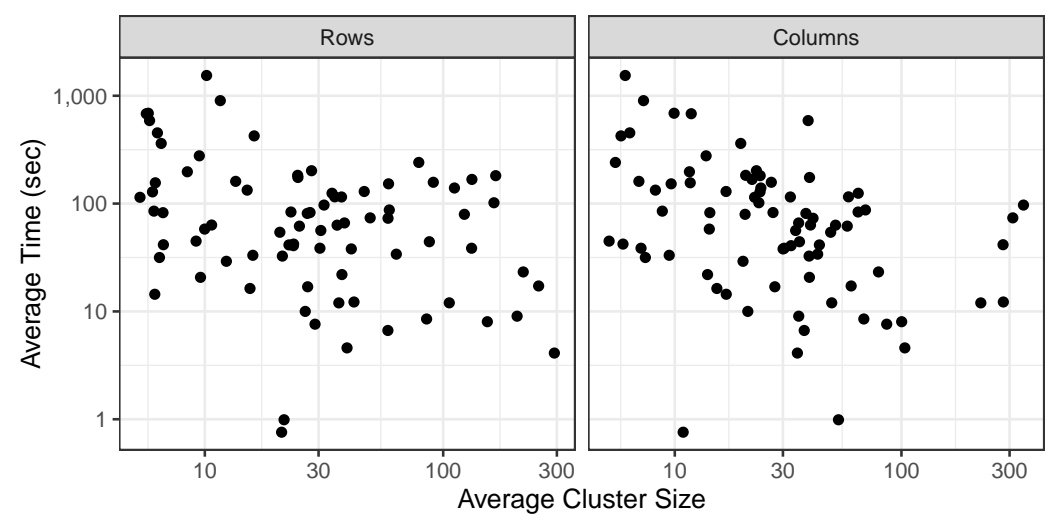

Figure 13: Relationship between average cluster sizes and elapsed time

\section{Acknowledgments}

This research was supported in part by Syngenta Seeds and by a Kingland Data Analytics Faculty Fellowship at Iowa State University.

\section{Bibliography}

S. Bergmann, J. Ihmels, and N. Barkai. Iterative signature algorithm for the analysis of large-scale gene expression data. Physical Review E - Statistical Physics, Plasmas, Fluids, and Related Interdisciplinary Topics, 2003. ISSN 1063651X. URL https://doi .org/10.1103/PhysRevE.67.031902. [p69]

S. Busygin, O. Prokopyev, and P. M. Pardalos. Biclustering in data mining. Computers and Operations Research, 35(9), 2008. URL https://doi.org/10.1016/j.cor. 2007.01.005. [p69]

Y. Cheng and G. M. Church. Biclustering of expression data. Proceedings International Conference on Intelligent Systems for Molecular Biology; ISMB. International Conference on Intelligent Systems for Molecular Biology, 8, 2000. [p69]

D. T. Ewoud. BiBitR: R Wrapper for Java Implementation of BiBit, 2017. R package version 0.4.2. [p69]

D. W. Goodall. A new similarity index based on probability. Biometrics, 22:882-907, 1966. [p71]

D. Gusenleitner and A. Culhane. iBBiG: Iterative Binary Biclustering of Genesets, 2019. URL http: //bcb.dfci.harvard.edu/ aedin/publications/. R package version 1.28.0. [p69]

F. M. Harper and J. A. Konstan. The MovieLens datasets. ACM Transactions on Interactive Intelligent Systems, 2015. [p81]

J. A. Hartigan. Direct clustering of a data matrix. Journal of the American Statistical Association, 67(337): 123-129, 1972. URL https://doi.org/10.1080/01621459.1972.10481214. [p69]

L. Hubert and P. Arabie. Comparing partitions. Journal of Classification, 1985. ISSN 01764268. doi: 10.1007/BF01908075. [p71]

S. Kaiser and F. Leisch. A toolbox for bicluster analysis in $\{\mathrm{R}\}$. Compstat 2008-Proceedings in Computational Statistics, pages 201-208, 2008. [p69]

T. Khamiakova. superbiclust: Generating Robust Biclusters from a Bicluster Set (Ensemble Biclustering), 2014. URL https://CRAN. R-project.org/package=superbiclust. R package version 1.1. [p69]

Y. Kluger, R. Basri, J. T. Chang, and M. Gerstein. Spectral biclustering of microarray data: Coclustering genes and conditions. Genome Research, 13(4):703-716, 2003. URL https://doi .org/10.1101/gr. 648603. [p69]

L. Lazzeroni and A. Owen. Plaid models for gene expression data. Statistica Sinica, 12:61-86, 2002. ISSN 10170405. URL https://doi .org/10.1017/CB09781107415324.004. [p69]

J. Li, J. Reisner, H. Pham, S. Olafsson, and S. Vardeman. Biclustering for missing data. Information Sciences, 510:304-316, 2020. URL https://doi.org/10.1016/j.ins.2019.09.047. [p70, 73, 82] 
M. Malosetti, J. M. Ribaut, and F. A. van Eeuwijk. The statistical analysis of multi-environment data: Modeling genotype-by-environment interaction and its genetic basis. Frontiers in Physiology, 4(44), 2013. URL https://doi.org/10.3389/fphys.2013.00044. [p79]

A. Oghabian, S. Kilpinen, S. Hautaniemi, and E. Czeizler. Biclustering methods: Biological relevance and application in gene expression analysis. PLOS ONE, 9(3):1-10, 03 2014. doi: 10.1371/journal. pone.0090801. URL https://doi.org/10.1371/journal.pone.0090801. [p81]

M. Rand. Objective criteria for the evaluation of methods clustering. Journal of the American Statistical Association, 66(336):846-850, 1971. URL https://doi .org/10.1080/01621459.1971.10482356. [p71]

M. Sill and S. Kaiser. s4vd: Biclustering via Sparse Singular Value Decomposition Incorporating Stability Selection, 2015. URL https: //CRAN. R-project. org/package=s4vd. R package version 1.1-1. [p69]

K. M. Tan and D. M. Witten. Sparse Biclustering of Transposable Data. Journal of Computational and Graphical Statistics, 2014. ISSN 15372715. URL https://doi .org/10.1080/10618600.2013.852554. [p69]

H. Wickham. ggplot2: Elegant Graphics for Data Analysis. Springer-Verlag New York, 2009. ISBN 978-0-387-98140-6. URL http://ggplot2.org. [p73]

H. Wickham. tidyverse: Easily Install and Load 'Tidyverse' Packages. 2016. URL https://cran.rproject.org/package=tidyverse. [p72]

H. Wickham. nycflights13: Flights that departed NYC in 2013, 2017. URL https: //CRAN. R-project.org/ package=nycflights13. R package version 0.2.2. [p72]

H. Wickham and L. Henry. tidyr: Easily Tidy Data with 'spread()' and 'gather()' Functions, 2019. URL https://CRAN. R-project. org/package=tidyr. R package version 0.8.3. [p79]

Y. Zhang, J. Xie, J. Yang, A. Fennell, C. Zhang, and Q. Ma. QUBIC: a bioconductor package for qualitative biclustering analysis of gene co-expression data. Bioinformatics, 33(3):450-452, 2017. URL https://doi.org/10.1093/bioinformatics/btw635. [p69]

\author{
John Reisner \\ Department of Statistics \\ Iowa State University \\ United States \\ johntreisner@gmail.com
}

Hieu Pham

Department of Industrial and Manufacturing Systems Engineering

Iowa State University

United States

htpham@iastate.edu

Sigurdur Olafsson

Department of Industrial and Manufacturing Systems Engineering

Iowa State University

United States

olafsson@iastate.edu

Stephen Vardeman

Department of Statistics

Department of Industrial and Manufacturing Systems Engineering

Iowa State University

United States

vardeman@iastate. edu

Jing $L i$

Boehringer Ingelheim Animal Health

St. Joseph, Missouri

United States

jingli2014cymailegmail.com 